\title{
Microwave Tomography for Biomedical Quantitative Imaging
}

\section{Sima Noghanian*}

Electrical Engineering Department, University of North Dakota, USA

The use of microwave to find information about an unknown object is not a new concept. There are numerous examples of applications of microwave in Non-Destructive Testing (NDT), however, very few of them are using Micro Wave Tomography (MWT). In MWT the unknown objects are localized and quantitatively categorized by measuring the scattered fields using an array of receivers surrounding the objects. These scattered fields are due to a known incident field, sent by one or several transmitter antennas. In the past two decades MWT has drawn attention as a quantitative imaging modality for biomedical applications such as breast cancer imaging, brain imaging, and lung cancer imaging. These applications are mostly of interest due to shortcomings of other imaging modalities.

$\mathrm{X}$-ray mammography is routinely used for breast screening. According to the American Cancer Society (ACS), breast cancer is the leading cause of cancer death among women worldwide [1]. In the United States of America, more than 40,000 women die annually from breast cancer. It is the second-leading cause of cancer death in females after lung cancer. Excluding cancers of the skin, breast cancer is the most common cancer diagnosed among U.S. women, accounting for more than one in four cancers that develop over time. One out of eight American women who live to be 85 years of age will develop breast cancer, a risk that was one out of 14 in 1960. In the United States, breast cancer is newly diagnosed every three minutes, and a woman will die from breast cancer every 13 minutes. Breast cancer is also the most invasive cancer among women in the U.S., accounting for nearly one out of every three cancers diagnosed. Early detection is vital to curing breast cancer. Mammography is the current "gold standard" for breast screening. However, mammography is not a sufficiently sensitive screening technique. Although mammography is highly sensitive in post-menopausal women, it has some shortcomings:

- This technique reveals only $56 \%$ of cancers in premenopausal women. Mammography has an overall $20 \%$ false negative rate in women under age 60 , and up to $40 \%$ false negative rate in women under age 50 , due to breast density.

- Mammography cannot detect an exponentially fast-growing cancer in the pre-invasive stage.

- Mammography has an overall 50\% false positive rate, which leads to unnecessary biopsies. It has been determined that $85 \%$ of mammography-initiated biopsies are negative.

- Mammography examinations cause discomfort due to breast compression.

Medical imaging methods such as Magnetic Resonance Imaging (MRI) can be used in some of these situations. MRI scans use magnets and radio waves to produce very detailed cross-sectional images of the body. The most useful MRI exams for breast imaging use a contrast material, gadolinium, that is injected into a vein in the arm before or during the exam. This improves the ability of MRI to clearly show breast tissue details. Although MRI is more sensitive in detecting cancers than mammograms, at times MRI can be too sensitive, picking up abnormalities that are not actually cancerous. In some cases, it has been questioned whether or not this leads to a woman undergoing more treatment than is necessary. There is still disagreement as to whether all women with early stage breast cancer should have this test performed [2].

The feasibility of using microwave for brain imaging has been investigated $[3,4]$. A majority of work is on stroke monitoring. A stroke occurs when the blood supply to part of the brain is suddenly interrupted, or when blood vessel in the brain bursts, spilling blood into the spaces surrounding brain cells. There are two types of stroke: Ischemic (in adequate blood flow due to blockage) and Hemorrhagic (bleeding into or around the brain). A Transient Ischemic Attack (TIA) is when a mini-stroke starts but resolves leaving no noticeable symptoms. Onethird of people who have had TIA will have an acute stroke sometime in the future. In addition recurrent stroke is frequent. $25 \%$ of people who recover from the first stroke have another one within 5 years. The risk of disability and death increases with each recurrence [5]. Each year, 795,000 people experience a new or recurrent stroke. Approximately 610,000 of these are first attacks, and 185,000 are recurrent attacks. Of all strokes, $87 \%$ are ischemic, $13 \%$ are hemorrhage. The incidence of TIA in the United States has been estimated to be 200,000 to 500,000 per year. On average, every 40 seconds, someone in the United States has a stroke. On average, every 4 minutes, someone dies of a stroke. Stroke accounted for 1 of every 18 deaths in the United States in 2006 [6]. Currently, the main tool used to detect stroke is Computerized Tomography (CT) and MRI. These are very effective tools, however, they are expensive and not every hospital is equipped with them. In addition, they are not portable and suitable for regular monitoring.

\section{Microwave Biomedical Tomography: Advantages and Challenges}

Microwave imaging has a great potential to become a complimentary imaging modality for biomedical applications such as cancer and stroke monitoring. Microwave imaging offers many attractive features, such as having a low health risk, being noninvasive, simple to perform, and cost effective, and having minimal discomfort. Microwave images are maps of the electrical property distributions of objects. Two parameters that describe the propagation of electromagnetic radiation within materials are dielectric permittivity and conductivity. These properties are related to other physical properties such as temperature, density, and water content. Low-water-content tissues such as skin and fat have permittivity values that are estimated to be less than half those of highwater-content tissues such as blood and cancerous tissues [7-14].

Biomedical quantitative imaging is facing many challenges before it becomes a practical imaging modality:

*Corresponding author: Sima Noghanian, Electrical Engineering Department, University of North Dakota, USA, Tel: 701-777-4433; E-mail: sima.noghanian@engr.und.edu

Received June 30, 2012; Accepted July 02, 2012; Published July 04, 2012

Citation: Noghanian S (2012) Microwave Tomography for Biomedical Quantitative Imaging. J Elec Electron 1:e107. doi:10.4172/2167-101X.1000e107

Copyright: (c) 2012 Noghanian S. This is an open-access article distributed under the terms of the Creative Commons Attribution License, which permits unrestricted use, distribution, and reproduction in any medium, provided the original author and source are credited. 
- Human body is an inhomogeneous object that varies in time. Any imaging method should consider the in homogeneity of the object.

- Tissues in general are very lossy. This limits the penetration depth of microwaves, especially at high frequencies.

- Microwave imaging is based on contrast in dielectric properties. While some tissues show a big contrast in dielectric properties (such as fat and cancer tissues), some do not have a large contrast.

- Inverse imaging can be very computational intensive; therefore, image reconstruction can be very slow.

- Microwave quantitative imaging is based on a priori knowledge of in vivo tissue dielectric properties. While there is a good literature on ex vivo tissue properties [7-14], there is very limited information about in vivo tissue properties.

- Field measurements should be very precise, especially in phase measurement and calibration.

- There are challenges to overcome in regards to modeling and calibration errors.

\section{Achievements}

Perhaps the recent advances in computational power and microwave hardware design are the reasons that many researchers are now considering MWT as a possible cost-effective frequent screening tool. Therefore, the research community has seen many achievements in the past decade to bring MWT closer to wide clinical use. These achievements can be categorized into two major groups: image reconstruction using inverse problem algorithms, and measurement setups.

Image reconstruction in MWT is based on solving an inverse scattering problem. This usually is treated as an optimization problem, where the error between the measured scattered electric fields and those computed by a numerical forward solver are being minimized. The resolution of the resulted images dictates the complexity of the numerical forward solver. The optimization search space depends on range of possible dielectric properties and tissue compositions. Therefore, a high resolution image with accurate dielectric properties is an intensive computational problem. In addition the problem is ill-posed and the solution might not be unique or exist at all. The illposedness of the problem is due to limited sampling points. In addition, the solution could be unstable, i.e. a small change in the incident field may cause large changes in the solution.

To overcome the ill-posedness, many effective regularization methods are proposed. Tikonov [15], Krylov [16], and Multiplicative Regularizations [17] are among some well-known ones used in local and deterministic optimization methods such as Gauss-Newton Inversion (GNI) [18]. They are also used for Contrast Source Imaging (CSI) which does not need a forward solver [19,20]. Some new regularization methods based on a priori information about human physiological structures are also suggested [21].

Inverse-scattering is a nonlinear problem that requires iterative methods to invert Maxwell's equations in order to reach a solution. If the changes in the dielectric properties are small, these equations can be linearized using different approximations such as the Born approximation [22]. In Born approximation it is assumed that scattering fields are weak in compare to the incident fields. Therefore, the field inside the object of interest is approximated by a known incident field. These approximate methods convert the problem to a linear system of equations that can be solved numerically. A regularization term must be used to stabilize the solution. Thus the problem can be solved by a local optimization method such as conjugate gradient [23]. While such methods are very effective when the object under the test is simple, in medical imaging with a high degree of heterogeneity and high dielectric permittivity contrast they can lead to non-real solutions. Iterative methods are suggested to improve these approximations. Born Iterative Method (BIM) and Distorted BIM (DBIM) are introduced which use distorted Green's function and update it during the iterative optimization $[24,25]$. There has been some study to extend these methods to three dimensional imaging [26-28].

In another set of solutions, instead of integral equations, Partial Differential Equation (PDE) are solved through numerical methods such as Finite Difference Time Domain (FDTD) or Finite Element Method (FEM) [29,30]. These methods can be combined with stochastic method and global optimization algorithms such as Particle Swarm Optimizations and Genetic Algorithms [31]. As expected these methods are more computationally intense than integral equation based methods.

In regards to measurement setups, a lot of attempts have been made to design efficient wideband and Ultra Wideband (UWB) antennas [32,34]. Various Two Dimensional (2D) and Three Dimensional (3D) setups are under development [35-39]. Some setups are directly using fields received by antennas and calibrate them to be used in inversion algorithms, and sum use probes to sample the field [40]. There are also attempt to have microwave imaging in conjunction with MRI systems [41].

\section{Discussion and Conclusion}

As we move forward toward better and more accessible screening tools for detecting and preventing fatal diseases such as cancer and stroke, MWT becomes more attractive to be used as a cost-effective monitoring modality. Although there is a debate if MWT can reach the desired resolution, one should keep in mind that there are many possibilities to improve the existing resolution. In many MWT inversion algorithms speed and accuracy can be increased by having a priori information. This makes MWT an ideal tool for subsequent screening after an initial X-ray or MRI. The information taken from X-ray and/ or MRI can be segmented and translated to physical dimensions of the object of interest and be used as an initial guess for optimization methods. In addition, information about tissue dielectric properties can be used in regularization terms to improve the chance of getting to the correct answer with less computation time. In MWT there is always a compromise between resolution, accuracy and runtime. By resolution we mean the pixel size of image and by accuracy we mean the correctness of estimated dielectric properties. As our computational power improves by introducing parallel algorithm on High Performance Computers (HPC), and Graphics Processing Units (GPU) in near future many of computational barriers will be removed and we can achieve the desired accuracy and resolution. Currently, the numerical models do not include antenna systems and the incident field is approximated by plane waves or line sources. Therefore, accuracy is heavily dependent on modeling and calibration. In future, when the computational power is available, we can include the antenna as part of forward simulations and remove many assumptions to increase the accuracy.

There is very little work done on the specificity and sensitivity of this method. Today many practical setups are getting closer to be used 
in clinical testing, therefore, it is expected that more information about MWT's specificity and sensitivity become available in near future.

In conclusion, although MWT might seem not be able to compete with existing screening and imaging modalities such as X-ray, CAT and MRI yet, in near future it will provide a good quantitative screening tool that has the potential to be cost-effective, portable and widely used.

\section{References}

1. http://www.cancer.org/Research/CancerFactsFigures/index

2. Tillman GF, Orel SG, Schnall MD, Schultz DJ, Tan JE, et al. (2002) Effect of Breast Magnetic Resonance Imaging on the Clinical Management of Women With Early-Stage Breast Carcinoma. J Clin Oncol 20: 3413-3423.

3. Semenov S (2009) Microwave tomography: review of the progress towards clinical applications. Philos Transact A Math Phys Eng Sci 367: 3021-3042.

4. Ireland D, Bialkowski ME (2011) Microwave head imaging for stroke detection. PIER M 21: 163-175.

5. Vital M "Stroke," Office of Communication and Public Liaison National Institute of Neurological Disorders and Stroke (NINDS).

6. Heart Disease and Stroke Statistics-2010 Update: A Report From the American Heart Association.

7. Chaudhary SS, Mishra RK, Swarup A, Thomas JM (1984) Dielectric properties of normal \& malignant human breast tissues at radiowave \& microwave frequencies. Indian J Biochem Biophys 21:76-79.

8. Gabriel C, Gabriel S, Corthout E (1996) The dielectric properties of biological tissues: I. Literature survey. Phys Med Biol 41: 2231-2249.

9. Gabriel S, Lau RW, Gabriel C (1996) The dielectric properties of biological tissues: II. Measurements in the frequency range $10 \mathrm{~Hz}$ to $20 \mathrm{GHz}$. Phys Med Biol 41: 2251-2269.

10. Gabriel S, Lau RW, Gabriel C (1996) The dielectric properties of biological tissues: III. Parametric models for the dielectric spectrum of tissues. Phys Med Biol 41: 2271-2293.

11. Gabriel C (1996) Compilation of the dielectric properties of body tissues at RF and microwave frequencies.

12. Joines WT, Zhang Y, Li C, Jirtle RL (1994) The measured electrical properties of normal and malignant human tissues from 50 to $900 \mathrm{MHz}$. Med Phys 21: 547-550.

13. Lazebnik M, McCartney L, Popovic D, Watkins CB, Lindstrom MJ, et al. (2007) A large-scale study of the ultrawideband microwave dielectric properties of normal breast tissue obtained from reduction surgeries. Phys Med Biol 52 2637-2656.

14. Lazebnik M, Popovic D, McCartney L, Watkins CB, Lindstrom MJ, et al. (2007) A large-scale study of the ultrawideband microwave dielectric properties of normal, benign, and malignant breast tissues obtained from cancer surgeries. Phys Med Biol 52: 6093-6115.

15. Tikhonov AN, Arsenin VY(1977) Solutions of ill-posed problems. John Wiley \& Sons, New York.

16. Hanke M (1995) Conjugate Gradient Type Methods for III-Posed Problems. Chapman and Hall/CRC.

17. Mojabi P, LoVetri J (2009) Overview and Classification of Some Regularization Techniques for the Gauss-Newton Inversion Method Applied to Inverse Scattering Problems. IEEE Trans Antennas Propag 57: 2658-2665.

18. Joachimowicz N, Pichot C, Hugonin JP (1991) Inverse scattering: an iterative numerical method for electromagnetic imaging. IEEE Trans Antennas Propag 39: $1742-1753$

19. Abubakar A, Van den Berg PM, Mallorqui JJ (2002) Imaging of biomedical data using a multiplicative regularized contrast source inversion method. IEEE Trans Microw Theory Tech 50: 1761-1771.

20. Gilmore C, Mojabi P, LoVetri J (2009) Comparison of an Enhanced Distorted Born Iterative Method and the Multiplicative-Regularized Contrast Source Inversion method. IEEE Trans Antennas Propag 57: 2341-2351.

21. Ashtari A, Noghanian S, Sabouni A, Aronsson J, Thomas G, et al. (2010)
Using a priori Information for Regularization in Breast Microwave Image Reconstruction. IEEE Trans Biomed Eng 57: 2197-2208.

22. Born M, Wolf E (1980) Principles of Optics, 6th edition, New York: Pergamon Press.

23. Kleinman RE, Van den Berg PM (1992) A modified gradient method for twodimensional problems in tomography. J Comput Appl Math 42: 17-35.

24. Chew WC, Wang YM (1990) Reconstruction of two-dimensional permittivity distribution using the distorted Born iterative method. IEEE Trans Med Imaging 9: 218-225.

25. Otto G, Chew WC (1994) Microwave inverse scattering - local shape function imaging for improved resolution of strong scatterers. IEEE Trans Microw Theory Tech 42: 137-141.

26. Bulyshev AE, Souvorov AE, Semenov SY, Posukh VG, Sizov YE (2004) Three-dimensional vector microwave tomography: theory and computational experiments. Inverse Probl 20: 1239-1259.

27. Abubakar A, Van den Berg PM (2004) Iterative forward and inverse algorithms based on domain integral equations for three-dimensional electric and magnetic objects. J Comput Phys 195: 236-262.

28. Zhang ZQ, Liu QH (2004) Three-dimensional nonlinear image reconstruction for microwave biomedical imaging. IEEE Trans Biomed Eng 51: 544-548.

29. Fhager A, Padhi SK, Howard J (2009) 3D Image Reconstruction in Microwave Tomography Using an Efficient FDTD Model. IEEE Antennas Wirel Propag Lett 8: 1353-1356.

30. Fang Q, Meaney PM, Paulsen KD (2004) Microwave image reconstruction of tissue property dispersion characteristics utilizing multiple-frequency information. IEEE Trans Microw Theory Tech 52: 1866-1875.

31. Sabouni A, Noghanian S, Pistorius S (2010) A global optimization technique for microwave imaging of the inhomogeneous and dispersive breast. Canadian Journal of Electrical and Computer Engineering 35: 15-24.

32. Bourqui J, Okoniewski M, Fear EC (2010) Balanced Antipodal Vivaldi Antenna With Dielectric Director for Near-Field Microwave Imaging. IEEE Trans Antennas Propag 58: 2318-2326.

33. Abbosh AM, Kan HK, Bialkowski ME (2006) Compact ultra-wideband planar tapered slot antenna for use in a microwave imaging system. Microw Opt Technol Lett 48: 2212-2216.

34. Ostadrahimi M, Noghanian S, Shafai L, Zakaria A, Kaye C, et al. (2010) Investigating a double layer Vivaldi antenna design for fixed array field measurement. International Journal of Ultra Wideband Communications and Systems 1: 282-290.

35. Gilmore C, Mojabi P, Zakaria A, Ostadrahimi M, Kaye C, et al (2010) A Wideband Microwave Tomography System With a Novel Frequency Selection Procedure. IEEE Trans Biomed Eng 57: 894-904.

36. Semenov SY, Svenson RH, Bulyshev AE, Souvorov AE, Nazarov AG, et al. (2000) Spatial resolution of microwave tomography for detection of myocardia ischemia and infarction-experimental study on two-dimensional models. IEEE Trans Microw Theory Tech 48: 538-544.

37. Meaney PM, Fanning MW, Raynolds T, Fox CJ, Fang Q, et al. (2007) Initia clinical experience with microwave breast imaging in women with normal mammography. Acad Radiol 14: 207-218.

38. Poplack SP, Tosteson TD, Wells WA, Pogue BW, Meaney PM, et al. (2007) Electromagnetic breast imaging: results of a pilot study in women with abnormal mammograms. Radiology 243: 350-359.

39. Bourqui J, Sill JM, Fear EC (2012) A prototype system for measuring microwave frequency reflections from the breast. Int J Biomed Imaging

40. Ostadrahimi M, Mojabi P, Noghanian S, Shafai L, Pistorius S, et al. (2012) A Novel Microwave Tomography System Based on the Scattering Probe Technique. IEEE Trans Instrum Meas 61: 379-390.

41. Aguilar SM, Al-Joumayly MA, Shea JD, Behdad N, Hagness SC (2011) Design of a microwave breast imaging array composed of dual-band miniaturized antennas. 2011 XXXth URSI General Assembly and Scientific Symposium: 1 - 